\title{
Autophagy is involved in aldosterone-induced mesangial cell proliferation
}

\author{
MIN YANG $^{1 *}$, BIN WANG $^{2 *}$, LIYING MIAO $^{1}$, XIANLIN XU $^{1}$ and XIAOZHOU HE ${ }^{1}$ \\ ${ }^{1}$ Department of Nephrology, The Third Affiliated Hospital of Soochow University, Changzhou, Jiangsu 213003; \\ ${ }^{2}$ Division of Nephrology, Huashan Hospital and Institute of Nephrology, Fudan University, Shanghai 200040, P.R. China
}

Received July 31, 2015; Accepted August 22, 2016

DOI: $10.3892 / \mathrm{mmr} .2016 .5807$

\begin{abstract}
The aim of the present study was to investigate whether autophagy is involved in aldosterone (Aldo)-induced mesangial cell (MC) proliferation. MCs were incubated with $10^{-7} \mathrm{M}$ Aldo for $24 \mathrm{~h}$. Proliferation of MCs, and the underlying mechanisms, were subsequently analyzed using $\left[{ }^{3} \mathrm{H}\right]$ thymidine assay, cell counting assay, western blotting and RNA interference (RNAi). Aldo was revealed to induce autophagy, as indicated by the increased conversion from microtubule-associated protein 1A/1B-light chain 3 (LC3)-I to LC3-II, the increased expression levels of autophagy-related gene 7 (Atg7) and the increased degradation of p62, which was accompanied by MC proliferation. Notably, pharmacological inhibition of autophagy or RNAi-mediated knockdown of Atg7 attenuated Aldo-induced MC proliferation, suggesting that autophagy was at least partially responsible for this effect. The results of the present study provided evidence that autophagy is critical for regulating Aldo-induced MC proliferation.
\end{abstract}

\section{Introduction}

Increasing evidence indicates that abnormal growth of glomerular mesangial cells (MCs) is an early event in various glomerular diseases $(1,2)$. The stimulation of MC proliferation in response to glomerular injury has been attributed to numerous factors, including high glucose levels (3), renin-angiotensin-aldosterone (Aldo) system (RAAS) overactivation (4) and platelet-derived growth factor (5). Aldo, which is considered to be a dependent risk factor for chronic kidney disease, has become a focus of investigations into the development and progression of these diseases $(6,7)$. However, the

Correspondence to: Dr Xiaozhou He, Department of Nephrology, The Third Affiliated Hospital of Soochow University, 185 Juqian Road, Changzhou, Jiangsu 213003, P.R. China

E-mail: clturtle@126.com

*Contributed equally

Key words: mesangial cells, aldosterone, autophagy, proliferation, autophagy-related gene 7 molecular mechanism underlying Aldo-induced MC proliferation remains to be fully elucidated.

Macroautophagy (hereafter referred to as autophagy) is an evolutionarily conserved catabolic mechanism that is responsible for the maintenance of energy homeostasis. Proteins encoded by autophagy-related genes (Atgs), including Atg5 and Atg7, are critical for the regulation of autophagy (8). The C-terminal carboxyl group of Atg12 is activated by the E1 enzyme Atg7 with consumption of ATP to form a thioester bond with its catalytic cysteine residue, and eventually attaches to the amino group of the lysine residue in Atg5 via an isopeptide bond (9). This process is critical for the formation of the autophagosomal membrane. During autophagy, there may be an increased conversion of microtubule-associated protein 1A/1B-light chain 3 (LC3)-I to LC3-II in mammalian cells. In addition, p62, a selective substrate of autophagy, may serve as a marker of autophagy (10). Autophagy, which contributes to the abnormal proliferation of cancer cells $(11,12)$, has also been implicated in the physiological and pathophysiological processes of numerous kidney diseases, including diabetic nephropathy (13), immunoglobulin A nephropathy (14) and aging kidney $(15,16)$.

The aim of the present study was to determine whether Aldo induced MC autophagy, and if so, to further examine the role of autophagy in Aldo-induced MC proliferation.

\section{Materials and methods}

Materials. The HBZY-1 rat MC line was obtained from the Chinese Center for Type Culture Collection (Wuhan, China). Chloroquine (CQ), 3-methyladenine (3-MA) and Aldo were purchased from Sigma-Aldrich; Merck Millipore (Darmstadt, Germany). Mouse anti- $\beta$-actin (catalog no. 3700), rabbit anti-Atg5 (catalog no. 12994), rabbit anti-Atg7 (catalog no. 8558), rabbit anti-LC3 (catalog no. 3868) and rabbit anti-p62 (catalog no. 5114) antibodies were purchased from Cell Signaling Technology, Inc. (Danvers, MA, USA). All other chemicals were of analytical grade.

Cell culture and treatment. Cells were cultured in Dulbecco's modified Eagle's medium (DMEM; Gibco; Thermo Fisher Scientific, Inc., Waltham, MA, USA) supplemented with $10 \%$ fetal calf serum (FCS; Sigma-Aldrich; Merck Millipore), $2 \mathrm{mM}$ glutamine, $100 \mathrm{U} / \mathrm{ml}$ penicillin and $100 \mathrm{mg} / \mathrm{ml}$ 
streptomycin, at $37^{\circ} \mathrm{C}$ in an atmosphere containing $5 \% \mathrm{CO}_{2}$. Following preincubation in serum-free DMEM for $24 \mathrm{~h}$, cells were treated with $10^{-7} \mathrm{M}$ Aldo for $24 \mathrm{~h}$ in DMEM containing $10 \%$ FCS in the presence or absence of $10 \mu \mathrm{M}$ CQ or $2 \mathrm{mM}$ 3-MA. Control group cells treated with an identical volume of PBS.

Cell proliferation assay. The $\left[{ }^{3} \mathrm{H}\right]$ thymidine assay was used as a qualitative index of DNA synthesis, as previously reported (17). Briefly, MCs were plated in 24-well plates and grown to $80 \%$ confluence. Cells were stimulated with $10^{-7} \mathrm{M}$ Aldo for $24 \mathrm{~h}$. Subsequently, $1 \mu \mathrm{Ci} / \mathrm{ml}\left[{ }^{3} \mathrm{H}\right]$ thymidine (PerkinElmer, Inc., Waltham, MA, USA) was added to each well for $3 \mathrm{~h}$. $\left[{ }^{3} \mathrm{H}\right]$ thymidine incorporation into trichloroacetic acid-insoluble material was then measured using a liquid scintillation spectrophotometer. To assess cell number, MCs were stimulated in 6-well plates with $10^{-7} \mathrm{M}$ Aldo for $24 \mathrm{~h}$ and counted using a Z1-Coulter Counter (Beckman Coulter, Inc., Brea, CA, USA).

Transient transfection of cells with small interfering (si) RNA. For knockdown experiments, MCs were transiently transfected with siRNA specifically targeting Atg7 or a negative control siRNA using Lipofectamine ${ }^{\circledR} 3000$ (Invitrogen; Thermo Fisher Scientific, Inc.) according to the manufacturer's protocol. Cells were transfected with 20 nM Atg7 siRNA (Santa Cruz Biotechnology, Inc., Dallas, TX, USA) or control siRNA (Santa Cruz Biotechnology, Inc.) for $24 \mathrm{~h}$ prior to further treatment. Cellular protein was extracted and subjected to Atg7 western blot analysis.

Western blotting. Total cellular proteins were extracted by lysing cells with buffer containing $150 \mathrm{mM} \mathrm{NaCl}, 0.1 \%$ Triton $\mathrm{X}-100,0.5 \%$ deoxycholate, $0.1 \%$ sodium dodecyl sulfate (SDS), $50 \mathrm{mM}$ Tris- $\mathrm{HCl}$ (pH 7.0) and $1 \mathrm{mM}$ ethylenediaminetetraacetic acid. Protein concentrations were determined using the bicinchoninic acid method (Beyotime Institute of Biotechnology, Haimen, China). MC protein extracts (50 $\mu \mathrm{g}$ /lane) were separated by $10 \%$ SDS-polyacrylamide gel electrophoresis and were transferred to nitrocellulose membranes. Following blocking with 5\% skimmed milk in Tris-buffered saline $(\mathrm{pH}$ 7.6) at room temperature for $1 \mathrm{~h}$, the membranes were incubated at $4{ }^{\circ} \mathrm{C}$ overnight with primary antibodies against LC3 (1:1,000), p62 (1:1,000), Atg7 (1:1,000) and $\beta$-actin (1:100). Membranes were then incubated with Alexa Fluor 790-conjugated goat anti-rabbit (1:2,500; catalog no. A27041; Thermo Fisher Scientific, Inc.) or Alexa Fluor 680-conjugated goat anti-mouse (1:5,000; catalog no. A28183; Thermo Fisher Scientific, Inc.) secondary antibodies, at room temperature for $1 \mathrm{~h}$. Protein bands were visualized with the LI-COR Odyssey ${ }^{\circledR}$ protein analysis system (LI-COR Biosciences, Lincoln, NE, USA). The relative intensity of each band was normalized to $\beta$-actin using Image $\mathrm{J}$ software version 1.48 (National Institutes of Health, Bethesda, MD, USA).

Statistical analysis. Quantitative data are presented as the mean \pm standard deviation. Statistical significance was determined using the unpaired Student's t-test or one-way analysis of variance followed by Tukey's post-hoc test. SPSS software version 13.0 (SPSS, Inc., Chicago, IL, USA) was used for statistical analysis. $\mathrm{P}<0.05$ was considered to indicate a statistically significant difference.

\section{Results}

Aldo induces MC proliferation. $\left[{ }^{3} \mathrm{H}\right]$ thymidine incorporation and cell counting assays were performed to evaluate $\mathrm{MC}$ proliferation. Compared with the control group, $10^{-7} \mathrm{M}$ Aldo significantly increased the proliferation of MCs $(\mathrm{P}<0.0001$; Fig. 1A and B).

Aldo induces MC autophagy. As presented in Fig. 2, incubation with Aldo (10-7 M) for $24 \mathrm{~h}$ resulted in an increased conversion from LC3-I to LC3-II $(\mathrm{P}=0.0003)$ and increased expression levels of Atg7 $(\mathrm{P}<0.0001)$ compared with the control group, as determined by western blotting. Furthermore, Aldo increased the degradation of a selective substrate of autophagy, p62 ( $\mathrm{P}=0.0017)$. However, Aldo had no significant effect on the protein expression levels of Atg5 (data not shown). These results suggest that Aldo treatment promoted cellular autophagy in MCs.

Pharmacological inhibition of autophagy prevents Aldo-induced MC proliferation. Numerous studies (18-20) have described the use of pharmacological agents to demonstrate the involvement of autophagy in the regulation of cell proliferation. 3-MA, which inhibits autophagosomal cargo-sequestration and CQ, which acts on lysosmal function were used to inhibit individual steps of the autophagic pathway. As presented in Fig. 3A and B, 3-MA blocked the conversion from LC3-I to LC3-II ( $\mathrm{P}<0.0001)$. Conversely, CQ increased the levels of LC3-II/LC3-I. The opposite effects of 3-MA and CQ on the level of LC3-II/LC3-I may be due to the fact that these two inhibitors block autophagy at different points $(\mathrm{P}<0.0001)$. In addition, 3-MA and CQ attenuated Aldo-induced $\mathrm{MC}$ proliferation $(\mathrm{P}<0.0001$; Fig. $3 \mathrm{C})$.

siRNA-mediated knockdown of Atg7 inhibits Aldo-induced $M C$ proliferation. To further support the finding that Aldo induced proliferation via enhancing autophagy, cells were depleted of Atg7 using RNA interference technology, to investigate its impact on Aldo-induced MC proliferation. Atg7 protein expression levels were assessed by western blot analysis. Atg7 $(\mathrm{P}<0.0001)$ and LC3-II/LC-I $(\mathrm{P}<0.0001)$ protein levels were markedly reduced in MCs transfected with siRNA-Atg7 compared with those of control MCs and MCs transfected with control siRNA (Fig. 4A and B). Furthermore, Atg7 silencing partially attenuated Aldo-induced MC proliferation $(\mathrm{P}<0.0001$; Fig. 4C).

\section{Discussion}

As a critical mediator of the RAAS, plasma and tissue Aldo levels are elevated in patients with hypertension (21), and diabetic and other progressive nephropathies $(22,23)$, and may exert various physiological effects (24). Although interruption of the RAAS with angiotensin-converting enzyme inhibitors and angiotensin receptor blockers significantly improves kidney injury, Aldo concentrations 'escape' to baseline during chronic therapy (25). Increasing attention has recently been 
A

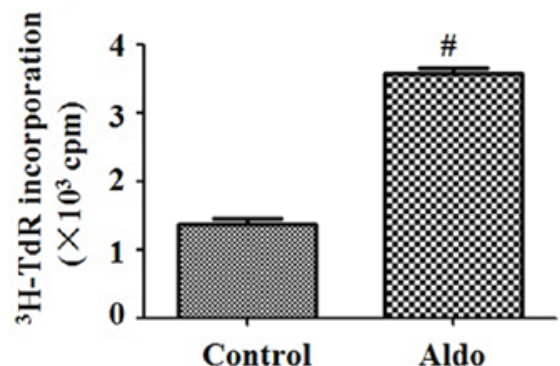

B

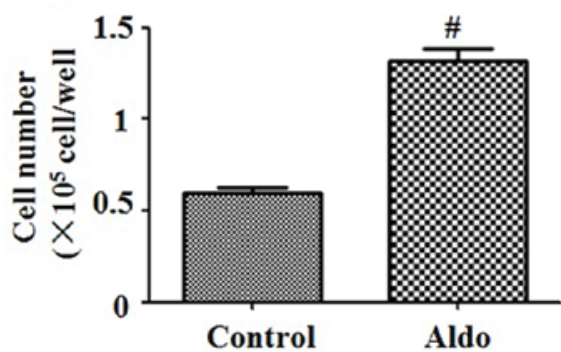

Figure 1. Aldo induced MC proliferation. MC proliferation was evaluated by (A) $\left[{ }^{3} \mathrm{H}\right]$ thymidine incorporation and (B) cell counting. MCs were treated with $10^{-7} \mathrm{M}$ Aldo for $24 \mathrm{~h}$. Data are presented as the mean \pm standard deviation of six series of experiments. ${ }^{~} \mathrm{P}<0.05$ vs. control. Aldo, aldosterone; MC, mesangial cell.
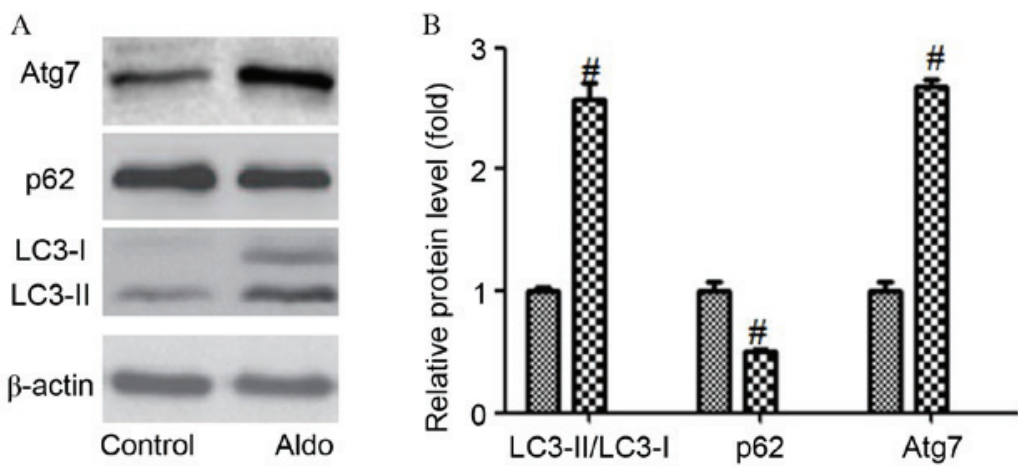

Figure 2. Aldo induced autophagy in MCs. (A) Western blot analysis revealed the protein expression levels of LC3-II/LC3-I, p62, Atg7 and $\beta$-actin following treatment with $10^{-7} \mathrm{M}$ Aldo for $24 \mathrm{~h}$. (B) Quantification of western blotting revealed the increase in LC3-II/LC3-I and Atg7 protein expression levels, and decrease in $\mathrm{p} 62$, following Aldo treatment. Results were normalized to $\beta$-actin. Data are presented as the mean \pm standard deviation of three series of experiments. " $\mathrm{P}<0.05$ vs. control. Aldo, aldosterone; MC, mesangial cell; LC3, microtubule-associated protein 1A/1B-light chain 3; Atg7, autophagy-related gene 7.

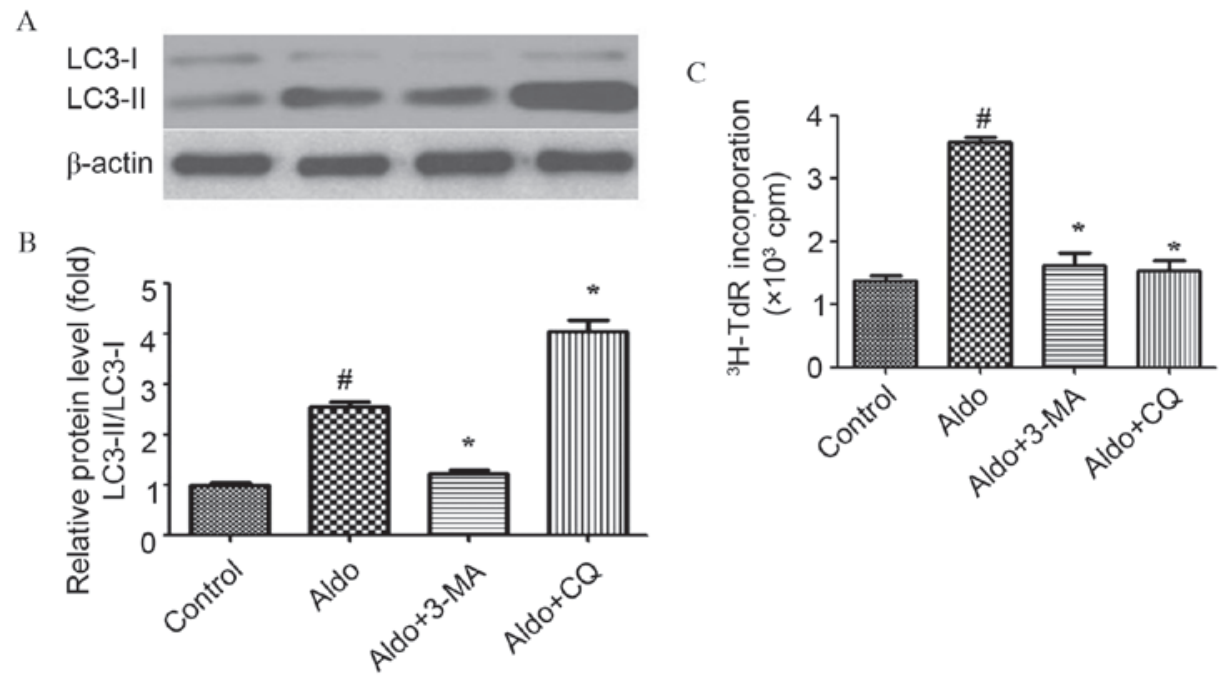

Figure 3. Pharmacologically inhibiting autophagy prevented Aldo-induced MC proliferation. (A) Western blot analysis revealed the protein expression levels of LC3-II/LC3-I and $\beta$-actin following treatment with $10^{-7} \mathrm{M}$ Aldo in the presence or absence of 3-MA or CQ for $24 \mathrm{~h}$. (B) Quantification of western blotting revealed the decrease of LC3-II/LC3-I protein expression levels following 3-MA treatment, and their increase following CQ treatment. Results were normalized to $\beta$-actin. Data are presented as the mean \pm standard deviation of three series of experiments. (C) MC proliferation was evaluated by $\left[{ }^{3} \mathrm{H}\right]$ thymidine incorporation following treatment with $10^{-7} \mathrm{M}$ Aldo in the presence or absence of 3-MA or CQ for $24 \mathrm{~h}$. Treatment with 3-MA or CQ abrogated Aldo-induced proliferation. Data are presented as the mean \pm standard deviation of six series of experiments. " $\mathrm{P}<0.05$ vs. control; " $\mathrm{P}<0.05$ vs. Aldo alone. Aldo, aldosterone; MC, mesangial cell; LC3, microtubule-associated protein 1A/1B-light chain 3; Atg7, autophagy-related gene 7; 3-MA, 3-methyladenine; CQ, chloroquine.

focused on the role of Aldo in the development and progression of kidney injury.
Elucidating the mechanisms underlying the induction of MC proliferation by Aldo may contribute to the 
A

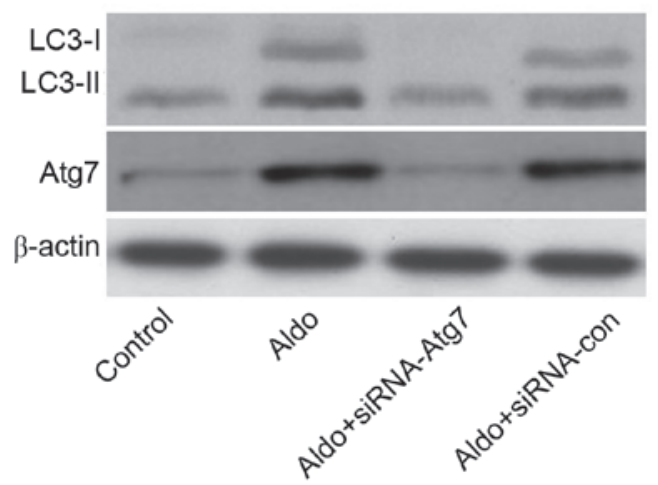

$\mathrm{C}$

B
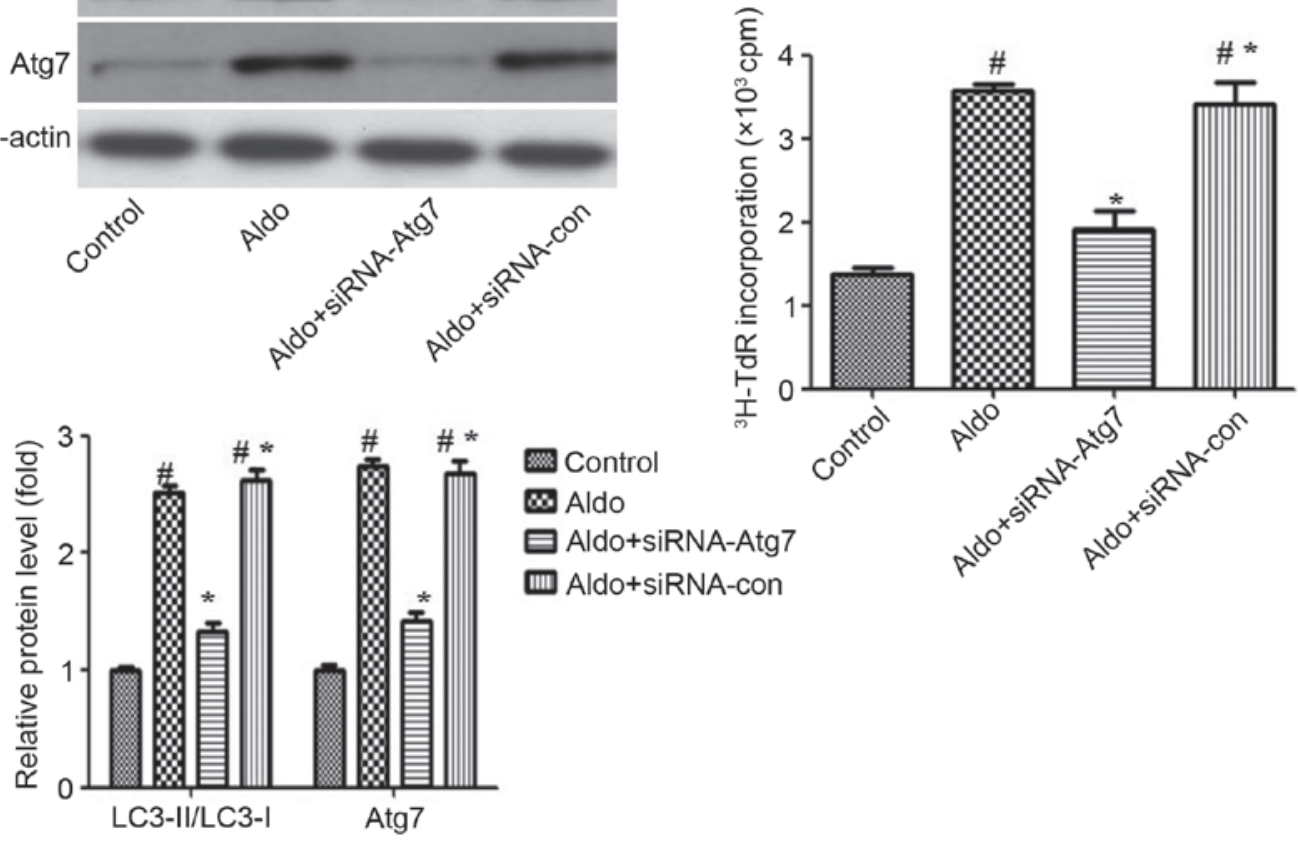

Figure 4. Inhibiting autophagy with siRNA-Atg7 prevented Aldo-induced MC proliferation. (A) MCs were transfected with $20 \mathrm{nM}$ siRNA-Atg7 or siRNA-con as a control. A total of $24 \mathrm{~h}$ post-transfection, MCs were treated with $10^{-7} \mathrm{M}$ Aldo for a further $24 \mathrm{~h}$. Western blot analysis revealed the protein expression levels of LC3-II/LC3-I, Atg7 and $\beta$-actin. (B) Quantification of western blotting revealed the decrease in LC3-II/LC3-I and Atg7 protein expression levels following siRNA-Atg7 transfection. Results were normalized to $\beta$-actin. Data are presented as the mean \pm standard deviation of three series of experiments. (C) MC proliferation was evaluated by $\left[{ }^{3} \mathrm{H}\right]$ thymidine incorporation following siRNA-Atg7 transfection. Transfection with siRNA-Atg7 abrogated Aldo-induced proliferation of MCs. Data are presented as the mean \pm standard deviation of six series of experiments. ${ }^{*} \mathrm{P}<0.05$ vs. control; " $\mathrm{P}<0.05$ vs. Aldo alone. siRNA, small interfering RNA; Atg7, autophagy-related gene 7; Aldo, aldosterone; MC, mesangial cell; con, control; LC3, microtubule-associated protein 1A/1B-light chain 3; con, control.

development of novel and effective treatment strategies for glomerular diseases. Huang et al (17) demonstrated that Aldo stimulated MC proliferation via the phosphoinositide 3-kinase/protein kinase B signaling pathway and reactive oxygen species-dependent epithelial growth factor receptor intracellular signaling. However, other, as yet unidentified, mechanisms may contribute to this process.

Autophagy is a self-protection mechanism that is triggered in response to harmful stimuli. A primary function of autophagy is to transport the surrounding materials and damaged organelles to lysosomes for degradation, thus enhancing intracellular recycling of degraded metabolites to maintain the energy metabolism cycle of cells. Autophagy fuels cancer cell metabolism (9), and contributes to abnormal proliferation (26), invasion (27), and resistance to chemotherapy and radiation therapy (28). In the present study, Aldo induced autophagy as indicated by the increased expression levels of Atg7, the increased conversion from LC3-I to LC3-II and the increased degradation of p62, which is accompanied by MC proliferation. Notably, pharmacological inhibition of autophagy or siRNA-mediated knockdown of Atg7 attenuated Aldo-induced MC proliferation, thus indicating that autophagy was at least partially responsible for this effect.

In conclusion, the results of the present study may partly explain the mechanism underlying the induction of abnormal MC proliferation by Aldo. The results suggested that autophagy may be a potential therapeutic target for the treatment of experimental mesangial proliferative renal disease. As the present study was performed in vitro, further in vivo studies are required to determine whether autophagy is involved in Aldo-induced MC proliferation in the kidney.

\section{Acknowledgements}

The present study was supported by a grant from the Basic Research Projects of Science and Technology Bureau of Changzhou City (grant no. CJ20140024).

\section{References}

1. Kurogi Y: Mesangial cell proliferation inhibitors for the treatment of proliferative glomerular disease. Med Res Rev 23: 15-31, 2003.

2. Feng Q, Huang S, Zhang A, Chen Q, Guo X, Chen R and Yang T: Y-box protein 1 stimulates mesangial cell proliferation via activation of ERK1/2. Nephron Exp Nephrol 113: e16-e25, 2009.

3. Lu X, Fan Q, Xu L, Li L, Yue Y, Xu Y, Su Y, Zhang D and Wang L: Ursolic acid attenuates diabetic mesangial cell injury through the up-regulation of autophagy via miRNA-21/PTEN/Akt/mTOR suppression. PLoS One 10: e117400, 2015.

4. Yuan Y, Zhang A, Huang S, Ding G and Chen R: A PPARgamma agonist inhibits aldosterone-induced mesangial cell proliferation by blocking ROS-dependent EGFR intracellular signaling. Am J Physiol Renal Physiol 300: F393-F402, 2011.

5. Wang B, Zhang A, Zheng J, Gong J, Li S, Zeng Z and Gan W: Bufalin inhibits platelet-derived growth factor-BB-induced mesangial cell proliferation through mediating cell cycle progression. Biol Pharm Bull 34: 967-973, 2011. 
6. Ajani AE, Marwick TH and Krum H: Aldosterone antagonists: A new treatment option for patients with post-myocardial infarction heart failure. Cardiovasc Revasc Med 7: 234-236, 2006.

7. Fiebeler A and Haller H: Participation of the mineralocorticoid receptor in cardiac and vascular remodeling. Nephron Physiol 94 p47-p50, 2003.

8. Mizushima N and Komatsu M: Autophagy: Renovation of cells and tissues. Cell 147: 728-741, 2011.

9. Mizushima N, Noda T, Yoshimori T, Tanaka Y, Ishii T, George MD, Klionsky DJ, Ohsumi M and Ohsumi Y: A protein conjugation system essential for autophagy. Nature 395: 395-398, 1998.

10. Wang B, Ding W, Zhang M, Li H, Guo H, Lin L, Chen J and $\mathrm{Gu}$ Y: Role of FOXO1 in aldosterone-induced autophagy: A compensatory protective mechanism related to podocyte injury. Oncotarget 26 May 2016, (Epub ahead of print).

11. White E: Deconvoluting the context-dependent role for autophagy in cancer. Nat Rev Cancer 12: 401-410, 2012.

12. Hu YL, Jahangiri A, Delay M and Aghi MK: Tumor cell autophagy as an adaptive response mediating resistance to treatments such as antiangiogenic therapy. Cancer Res 72: 4294-4299, 2012.

13. Xiao T, Guan X, Nie L, Wang S, Sun L, He T, Huang Y, Zhang J, Yang K, Wang J and Zhao J: Rapamycin promotes podocyte autophagy and ameliorates renal injury in diabetic mice. Mol Cell Biochem 394: 145-154, 2014.

14. Sato S, Yanagihara T, Ghazizadeh M, Ishizaki M, Adachi A, Sasaki Y, Igarashi T and Fukunaga Y: Correlation of autophagy type in podocytes with histopathological diagnosis of IgA nephropathy. Pathobiology 76: 221-226, 2009.

15. Hartleben B, Gödel M, Meyer-Schwesinger C, Liu S, Ulrich T, Köbler S, Wiech T, Grahammer F, Arnold SJ, Lindenmeyer MT, et al: Autophagy influences glomerular disease susceptibility and maintains podocyte homeostasis in aging mice. J Clin Invest 120: 1084-1096, 2010.

16. Kume S, Uzu T, Horiike K, Chin-Kanasaki M, Isshiki K, Araki S, Sugimoto T, Haneda M, Kashiwagi A and Koya D: Calorie restriction enhances cell adaptation to hypoxia through Sirtl-dependent mitochondrial autophagy in mouse aged kidney. J Clin Invest 120: 1043-1055, 2010.

17. Huang S, Zhang A, Ding G and Chen R: Aldosterone-induced mesangial cell proliferation is mediated by EGF receptor transactivation. Am J Physiol Renal Physiol 296: F1323-F1333, 2009.
18. Zhang H, Chen Z, Neelapu SS, Romaguera J and McCarty N: Hedgehog inhibitors selectively target cell migration and adhesion of mantle cell lymphoma in bone marrow microenvironment. Oncotarget 7: 14350-14365, 2016.

19. Button RW, Vincent JH, Strang CJ and Luo S: Dual PI-3 kinase/mTOR inhibition impairs autophagy flux and induces cell death independent of apoptosis and necroptosis. Oncotarget 7: 5157-5175, 2016.

20. Kowalik MA, Perra A, Ledda-Columbano GM, Ippolito G, Piacentini M, Columbano A and Falasca L: Induction of autophagy promotes the growth of early preneoplastic rat liver nodules. Oncotarget 7: 5788-5799, 2016.

21. Jugdutt BI: Expanding saga of the Renin-Angiotensin system: The angiotensin II Counter-Regulatory AT2 receptor pathway. Circulation 131: 1380-1383, 2015.

22. Mavrakanas TA, Gariani K and Martin PY: Mineralocorticoid receptor blockade in addition to angiotensin converting enzyme inhibitor or angiotensin II receptor blocker treatment: An emerging paradigm in diabetic nephropathy: A systematic review. Eur J Intern Med 25: 173-176, 2014

23. Rüster C and Wolf G: Renin-angiotensin-aldosterone system and progression of renal disease. J Am Soc Nephrol 17: 2985-2991, 2006.

24. Boldyreff B and Wehling M: Non-genomic actions of aldosterone: Mechanisms and consequences in kidney cells. Nephro Dial Transplant 18: 1693-1695, 2003.

25. Schrier RW, Masoumi A and Elhassan E: Aldosterone: Role in edematous disorders, hypertension, chronic renal failure, and metabolic syndrome. Clin J Am Soc Nephrol 5: 1132-1140, 2010.

26. Lee WY, Hsu KF, Chiang TA and Chen CJ: Phellinus linteus extract induces autophagy and synergizes with 5-fluorouracil to inhibit breast cancer cell growth. Nutr Cancer 67: 275-284, 2015.

27. Zhang W, Li Q, Song C and Lao L: Knockdown of autophagy-related protein 6 , Beclin-1, decreases cell growth, invasion, and metastasis and has a positive effect on chemotherapy-induced cytotoxicity in osteosarcoma cells. Tumour Biol 36: 2531-2539, 2015.

28. Pennati M, Lopergolo A, Profumo V, De Cesare M, Sbarra S, Valdagni R, Zaffaroni N, Gandellini P and Folini M: MiR-205 impairs the autophagic flux and enhances cisplatin cytotoxicity in castration-resistant prostate cancer cells. Biochem Pharmacol 87: 579-597, 2014. 